DOI: 10.1007/s00350-010-2763-5

\section{Unwirksame Vorausverfügungen des Arztes über Ansprüche gegen die ärztliche Abrechnungsstelle}

InsO $\S 35$ Abs. 2, 91 Abs. 1, 114 Abs. 1

Vorausverfügungen des Schuldners über Ansprüche, die sich gegen eine ärztliche Abrechnungsstelle richten, sind für die Zeit nach Verfahrenseröffnung auch nach Einführung des $₫ 35$ Abs. 2 InsO unwirksam, sofern der Verwalter die Arztpraxis fortführt (Bestätigung von BGHZ 167, 363).

BGH, Beschl. v. 18.2.2010 - IX ZR 61/09 (KG)

Problemstellung: Der BGH hatte sich in seiner Beschwerde gegen die Nichtzulassung der Revision lediglich mit der Frage zu befassen, ob die durch das Gesetz zur Vereinfachung des Insolvenzverfahrens vom 13.4.2007 neu hinzugefügten Abs. 2 und $3 \mathrm{zu} \ 35$ InsO, wonach der Insolvenzverwalter die selbstständige Erwerbstätigkeit in der Insolvenz natürlicher Personen freigeben kann, zur Änderung seiner Rechtsprechung zur Vorausabtretung von Ansprüchen gegen die Kassenärztliche Vereinigung führen. Bereits durch Urteil v. 11.5.2006 - IX ZR 247/03 - hatte der IX. Zivilsenat in einem Grundsatzurteil entschieden, dass die Abtretung oder Verpfändung von Forderungen auf Vergütung gegen die Kassenärztliche Vereinigung durch einen Arzt unwirksam ist, soweit sie sich auf Ansprüche bezieht, die auf nach Eröffnung des Insolvenzverfahrens erbrachten ärztlichen Leistungen beruhen (BGHZ 167, 363 ff.). Der Senat sieht keine Veranlassung, wegen der Neuregelung in $\$ 35$ Abs. 2 und 3 InsO von seiner früheren Rechtsprechung abzuweichen, auch wenn es gegen das Urteil vereinzelt Kritik gegeben habe (so z. B. Löwisch/Kaspers, in: MüKo/InsO, 2. Aufl., \114, Rdnr. 4; anders auch Uhlenbruck, ZVI 2002, 49 ff.). Den von der Nichtzulassungsbeschwerdebegründung hergestellten Zusammenhang zwischen der Regelung des $₫ 35$ Abs. 2 InsO und der der $\iint 91,114$ Abs. 1 InsO gebe es nicht. Soweit es um Ansprüche gegen die Kassenärztliche Vereinigung geht, handelt es sich nach Feststellung des Senats nicht um Bezüge aus einem privatrechtlichen Dienstverhältnis, sondern um Leistungen aus einem öffentlich-rechtlichen Rechtsverhältnis. Seitens der Kassenärztlichen/ Kassenzahnärztlichen Vereinigungen geleistete Zahlungen stellen keine Bezüge für Dienstleistungen dar, da die Behandlungsverträge nur mit den Patienten, nicht aber zugleich auch mit den Vereinigungen geschlossen wurden (vgl. auch Ries, ZInsO 2003, 1079 ff.). Der BGH sieht Ansprüche aus privatärztlichen Behandlungsverträgen nicht als ,,fortlaufende Bezüge aus einem Dienstverhältnis“i. S. von $\$ 114$ Abs. 1 InsO an, wenn er auch die Ansprüche eines Kassenarztes gegen die für ihn zuständige Kassenärztliche Vereinigung als „Arbeitseinkommen“ i.S. von $\$ 850$ Abs. 2 ZPO eingeordnet hat (BGHZ 96, 324, 326). Die Einrichtung einer Praxis ist nicht billig. Ein Arzt benötigt zunehmend Fremdkapital, das sich allerdings nach der Rechtsprechung des BGH nicht mehr durch Abtretung der Ansprüche auf sein Vertragsarzthonorar an die finanzierende Bank absichern lässt. Damit ist eine sichere Kreditgrundlage für

Bearbeitet von Prof. Dr. iur. Wilhelm Uhlenbruck,

Friedrich-Schmidt-Straße 39, 50931 Köln, Deutschland den Arzt weggefallen. Die Kreditinstitute haben entsprechend reagiert.

Zum Sachverhalt: Da es sich um eine Nichtzulassungsbeschwerde handelt, ist der der Entscheidung zugrunde liegende Sachverhalt nicht mitgeteilt. Jedoch ist davon auszugehen, dass über das Vermögen eines in eigener Praxis tätigen Arztes das Insolvenzverfahren eröffnet worden ist. Dieser hatte der kreditierenden Bank alle gegenwärtigen und zukünftigen Ansprüche gegen die für ihn zuständige Kassenärztliche Vereinigung sicherheitshalber abgetreten. Letztlich geht es um die Rechtsfrage, ob gem. \$114 Abs. 1 S. 1 InsO die Abtretung der Ansprüche für die Zeit vor Ablauf von zwei Jahren nach dem Ende des zurzeit der Eröffnung des Verfahrens laufenden Kalendermonats wirksam ist. Diese Frage hat der BGH wiederum verneint.

Aus den Gründen: [1] Die Nichtzulassungsbeschwerde ist statthaft ( 5544 Abs. 1 S. 1 ZPO) und zulässig ( 5544 Abs. 1 S. 2, Abs. 2 ZPO). Sie hat jedoch keinen Erfolg, weil sie keinen Zulassungsgrund nach $₫ 543$ Abs. 2 S. 1 ZPO aufdeckt.

[2] 1. Die von der Nichtzulassungsbeschwerde für rechtsgrundsätzlich gehaltene Frage, ob die Unwirksamkeit von Verfügungen, durch die der Schuldner Forderungen auf Vergütung von ärztlichen Leistungen abgetreten oder verpfändet hat, die auf nach Eröffnung des Insolvenzverfahrens erbrachten ärztlichen Leistungen beruhen (BGHZ 167, 363), auch nach Inkrafttreten des $₫ 35$ Abs. 2 und 3 InsO weiter gilt, rechtfertigt nicht die Zulassung der Revision. Zweck des $₫ 35$ Abs. 2 Ins $\mathrm{O}$ ist es, dem Insolvenzverwalter die Möglichkeit zu eröffnen, eine für die Masse verlustbringende Betriebsfortführung an den Schuldner freizugeben (BT-Dr. 16/3227, S. 17). Ist die Tätigkeit ertragreich, soll er sie mit der Masse fortführen können. Den Schutz von Zessionaren, denen über eine Vorausabtretung Forderungen des Schuldners aus der Zeit nach Verfahrenseröffnung abgetreten sind, bezweckt die Regelung nicht. Den von der Nichtzulassungsbeschwerdebegründung hergestellten $\mathrm{Zu}-$ sammenhang zwischen der Regelung des $\$ 35$ Abs. 2 InsO und der der $\$ \int 91,114$ Abs. 1 InsO gibt es nicht. Die gegenteilige Sicht hätte zur Folge, dass sich der Verwalter ohne Rücksicht auf den wirtschaftlichen Erfolg der Tätigkeit des Schuldners für eine Freigabe entscheiden müsste, weil er anderenfalls die Fortführung finanzieren müsste, ohne - jedenfalls für die Dauer von zwei Jahren - die Gegenleistung zur Masse ziehen zu können.

[3] 2. Die Entscheidung BGHZ 167, 363 ist in Rechtsprechung und Schrifttum auf breite Zustimmung gestoßen (vgl. OLG Düsseldorf, ZVI 2008, 429; LG Mosbach, ZInsO 2009, 198, 200; Bräuer, InvO 2006, 413, 416; Ries, ZVI 2007, 398, 399; Runkel, ZVI 2007, 45, 51; Linck, in: HKInsO, 5. Aufl., \$114, Rdnr. 3; Ahrendt, in: HmbKommInsO, 3. Aufl., \$114, Rdnr. 3; Moll, in: Kübler/Prütting/ Bork [Hrsg.], \$114, Rdnrn. 19f.). Soweit es an dem Urteil vereinzelte Kritik gegeben hat (Löwisch/Caspers, in: MüKo/InsO, 2. Aufl., \114, Rdnr. 4), sieht der Senat keinen Anlass, sich mit der Rechtsfrage erneut zu befassen. Der Grundgedanke der Entscheidung, dass die Erträge einer auf Kosten der Masse durchgeführten Betriebsfortführung nicht einem einzelnen Zessionar zufließen dürfen, gilt ungeachtet des Hinweises auf mögliche Schwierigkeiten der Angehörigen freier Berufe, Betriebsmittelkredite zu erlangen. Die Anerkennung des Absonderungsrechts nach Verfahrenseröffnung würde nur dazu führen, dass die Fortführung der Praxis sofort beendet werden müsste (vgl. Bräuer, a.a.O., S. 416).

[4] Im Übrigen wird von einer Begründung gemäß \544 Abs. 4 S. 2 Halbs. 2 ZPO abgesehen. 